\title{
The comparison of the lengths and diameters of main bronchi measured from two-dimensional and three-dimensional images in the same patients
}

\author{
Jeong Woo Lee, Ji-Seon Son, Jin-Wook Choi, Young-Jin Han, and Jun-Rae Lee \\ Department of Anesthesiology and Pain Medicine, Chonbuk National University Medical School and Hospital, Jeonju, Korea
}

Background: Recently, multi-planar reconstruction of the three-dimensional (3D) spiral chest CT scan has demonstrated superiority in the evaluation of the tracheobronchial tree. The goal of this study was to measure the lengths of the right and left main bronchi and their anteroposterior (AP) and transverse (TR) diameters using the 3D and two-dimensional (2D) images in the same adult respectively, and to evaluate the degree of correlation between them.

Methods: We measured the lengths of the right and left main bronchi from the carina to the first of their branches and the AP and TR diameters at the mid-portion of the right main bronchus and $2 \mathrm{~cm}$ below the carina in the left main bronchus. We determined the size of the left-sided double-lumen tube (DLT) based on the measured AP diameter of the left main bronchus from the 3D and 2D images, respectively.

Results: There was moderate correlation between the lengths of both main bronchi obtained from the 3D images and the $2 \mathrm{D}$ images, and between the AP diameter of the left main bronchus obtained from the 3D images and the 2D images. Same sized DLTs were estimated in $69 \%$ of the men and $34 \%$ of the women.

Conclusions: The lengths of the right and left main bronchi and their AP and TR diameters obtained from 3D images were not strongly correlated with those from 2D images. Therefore, a further study is needed to verify the superiority of 3D images in selecting the appropriate size of left-sided DLT. (Korean J Anesthesiol 2014; 66: 189-194)

Key Words: Bronchi, Computed tomography, Diameter.

\footnotetext{
Received: June 3, 2013. Revised: 1st, August 10, 2013; 2nd, October 2, 2013; 3rd, October 6, 2013. Accepted: October 7, 2013. Corresponding author: Ji-Seon Son, M.D., Department of Anesthesiology and Pain Medicine, Chonbuk National University Medical School and Hospital, 634-18, Keumam-dong, Jeonju 561-180, Korea. Tel: 82-63-250-1241, Fax: 82-63-250-1240, E-mail: sjs6803@jbnu.ac.kr It was presented, in part, at The 88th Annual Meeting of the Korean Society of Anesthesiologists, November 2011, Grand Hilton hotel, Seoul, Korea. (c) This is an open-access article distributed under the terms of the Creative Commons Attribution Non-Commercial License (http:// creativecommons.org/licenses/by-nc/3.0/), which permits unrestricted non-commercial use, distribution, and reproduction in any medium, provided the original work is properly cited.
} 


\section{Introduction}

The anesthetic management for cardiothoracic surgery has often used the double-lumen tube (DLT). If an anesthesiologist chooses an inappropriate DLT size, an air leak can occur when using an undersized DLT, and the bronchus can be injured when an oversized DLT [1,2]. Therefore, choosing an appropriately sized DLT is very important, and this becomes possible if we know the bronchial diameter in patients and the outer diameter of the DLT bronchial tip.

There is no currently used standard modality for estimating the length and diameter of the bronchial lumen. Studies on the method of measuring the length and diameter of the main bronchi have been attempted [3-9]. A method which may be easy and less harmful to the patient is the radiologic method such as a simple chest X-ray (CXR), computerized tomography (CT), and magnetic resonance imaging (MRI). Although the capabilities of CT and MRI have been extended to include assessment of the tracheobronchial tree $[4,5,9]$, the images of the bronchial lumen appear as elongated shapes in the two-dimensional (2D) images because the main bronchus is in an oblique orientation through the horizontal plane [4].

However, multi-planar reconstruction (MPR) of the spiral chest CT scan provides three-dimensional (3D) images from combining the 2D images, and the angle of the bronchial structure can be adjusted on 3D images. This technique has been used recently to obtain the most accurate measurements of the length and diameter of the main bronchi $[10,11]$. In clinical practice, a 3D spiral chest CT scan is not performed as part of the evaluation of pulmonary parenchymal diseases except for bronchial lesions because it is not superior to $2 \mathrm{D}$ spiral chest CT in diagnosing pulmonary parenchymal disease, and additionally, it costs more.

The goal of this study was to measure the length and the diameter of the main bronchus using the $3 \mathrm{D}$ and $2 \mathrm{D}$ images in the same adult patients, respectively, and to evaluate the degree of correlation between them.

\section{Materials and Methods}

We obtained written informed consent for all patients after Institutional Ethics Review Board approval. We conducted the current prospective study in 160 adults, comprising 80 men and 80 women, aged between 21 and 78 years old who were scheduled to undergo a spiral chest CT scan for medical diagnosis or health screening. The obtained sample size was 67 based on $90 \%$ statistical power and a minimal correlation coefficient of 0.4 collected in the pilot study. We intended to investigate different properties in the men and women. We wanted to exclude patients showing airway pathology such as carina or main bron- chus tumors or extrinsic compression of the main bronchi in the present study. Demographic data were recorded such as the age, height, weight, and sex.

Spiral chest CT scanning was performed for all patients during a normal end-inspiratory breath hold. The following homogeneous technical parameters were used for the definition dual-source computed tomography (DSCT; SIEMENS Medical Systems, Erlangen, Germany): collimation was $0.75 \times 16 \mathrm{~mm}$ and acquisition time was 7 seconds. The data were transferred to a stand-alone workstation (Syngo ${ }^{\mathrm{TM}}$; SIEMENS Medical Systems, Erlangen, Germany) to reconstruct images of 1-mm thickness at intervals of $1 \mathrm{~mm}$. After measuring the 3D images, the image data was converted into $2 \mathrm{D}$ images using conventional radiologic methods. To minimize measurement errors, all of the measurements were performed twice on the $200 \%$ enlarged image and the values were averaged.

On the 3D images, we could get the desired slice and adjust the angle freely. A single anesthesiologist trained by the radiologist attempted to obtain strictly orthogonal slices of the right and left main bronchial axes, as shown in Fig. 1. We measured the lengths of the main bronchi from the carina to the line which was right angles to the bronchial wall at the branches starting from the upper lobe. We measured the diameters (anteroposterior, AP; transverse, TR) of the mid-portion of the right main bronchus, and the diameters (AP, TR) of $2 \mathrm{~cm}$ below the carina of the left main bronchus because the bronchial cuff of the leftsided DLT often has its highest diameter $2 \mathrm{~cm}$ distal from the carina in conventional use.

On the 2D images, the measurements of length of the main bronchi were performed from the carina to the line, which was a right angle to the bronchial wall at the branches starting from the upper lobe. The measurement was performed over some slices in a way that overlapped on 2D images, if this was necessary. The measurements of the diameters (AP, TR) of the main bronchi were performed on the axial images through the midpoint of the inner line, which was at right angles to the bronchial wall $2 \mathrm{~cm}$ below the carina of the left main bronchus or half-way along the right main bronchus, respectively.

After collecting the data, we examined whether there was a significant correlation between the measured values on the $3 \mathrm{D}$ and $2 \mathrm{D}$ images. We also obtained the median and range of differences in values by subtracting values measured on the $2 \mathrm{D}$ images from the values measured on the 3D images. Incidentally, we determined the size of the left-sided DLT (BronchoCath ${ }^{\mathrm{TM}}$, Mallinckrodt, St. Louis, MO, USA) based on the measured AP diameter at $2 \mathrm{~cm}$ below the carina of the left main bronchus from the $3 \mathrm{D}$ and $2 \mathrm{D}$ images, respectively. The outer diameters of the bronchial lumen of the left-sided DLTs (BronchoCath ${ }^{\mathrm{TM}}$, Mallinckrodt, St. Louis, MO, USA) were reported as $8.7 \mathrm{~mm}$ for $32 \mathrm{Fr}, 9.6 \mathrm{~mm}$ for $35 \mathrm{Fr}, 10.2 \mathrm{~mm}$ for $37 \mathrm{Fr}, 10.7 \mathrm{~mm}$ for $39 \mathrm{Fr}$, 

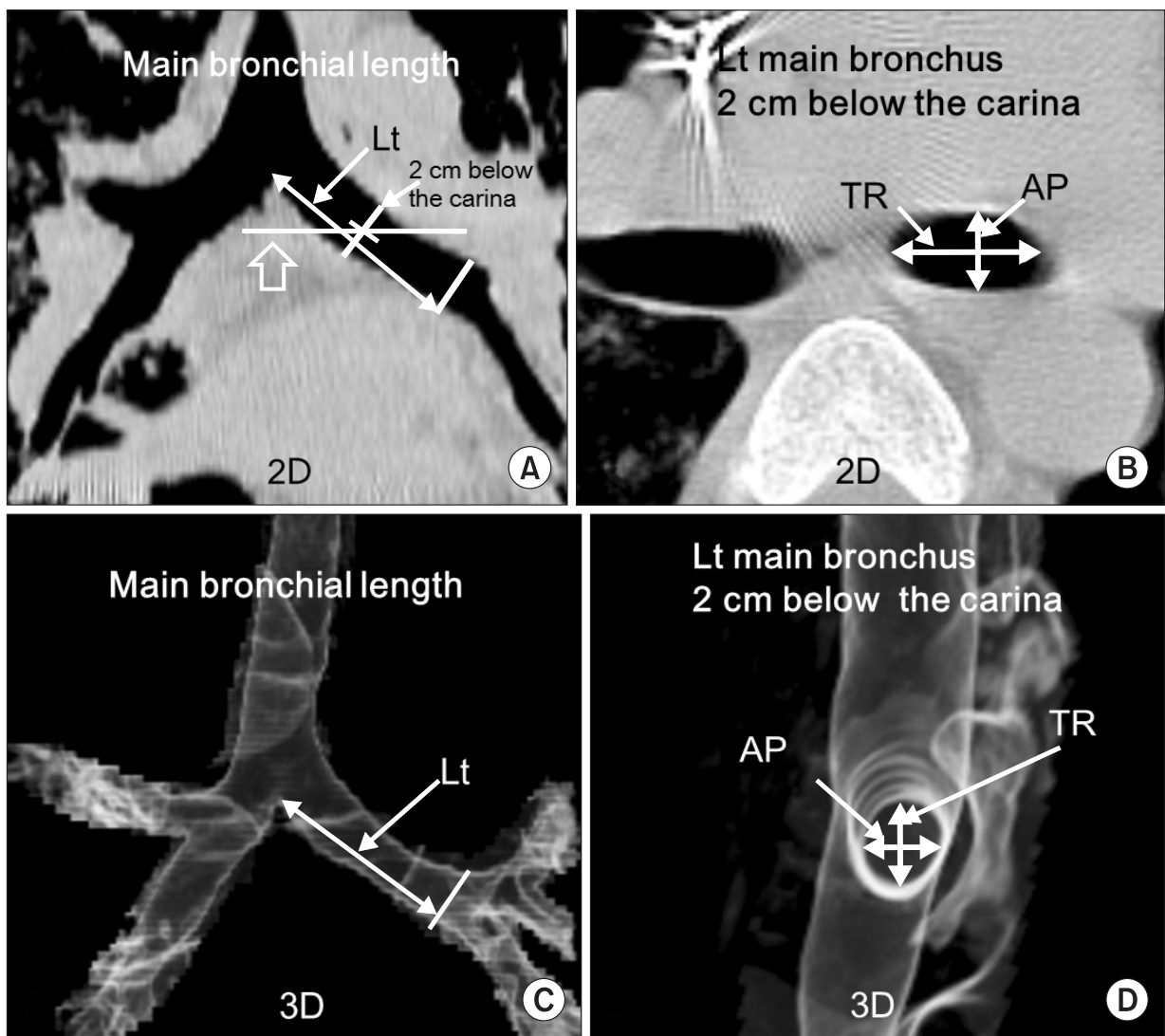

Fig. 1. The measurement of the length and diameter of the left main bronchus on the two-dimensional (2D) CT scan and the three-dimensional (3D) CT scan by multi-planar reconstruction. Any declinations in the $3 \mathrm{D}$ plane were adjusted to obtain a strictly orthogonal cut of the left main bronchial axis in the multi-planar reconstruction view. The length of the main bronchi was measured from the carina to the branches, starting from the upper lobe. In addition, the diameter of the left main bronchus was measured at $2 \mathrm{~cm}$ distal from the carina. (A) left main bronchus on the coronal image of 2D CT scan, (B) left main bronchus, $2 \mathrm{~cm}$ below the carina on the axial image of 2D CT scan, (C) left main bronchus on the adjusted 3D image, (D) left main bronchus, $2 \mathrm{~cm}$ below the carina on the adjusted 3D image. and $11.2 \mathrm{~mm}$ for $41 \mathrm{Fr}$, respectively [5].

The data were given as the mean (SD) or median (range) using descriptive statistics. The unpaired Student's t-test was used to test significant differences of the demographic data between men and women. Linear regression analysis was used to evaluate how the length and diameter of the bronchus on the 3D images related to those on the $2 \mathrm{D}$ images. A SigmaPlot for windows version 12.0 (Systat Software, Inc., San Joes, CA, USA) was used for all the analyses. $\mathrm{P}$ values less than 0.05 were considered statistically significant.

\section{Results}

The data of 160 patients ( 80 women and 80 men) were analyzed. Height and weight differed according to sex (Table 1). The lengths and diameters of the main bronchi were described in Tables 2 and 3. The measured mean lengths of the left main bronchus on 3D images were longer than those on the 2D images in men and women, but the measured mean lengths of the right main bronchus on $3 \mathrm{D}$ images were shorter than those on 2D images in women. The measured mean AP diameters of the main bronchi on 3D images were longer than those on $2 \mathrm{D}$ images. The measured mean TR diameters of the main bronchi
Table 1. Study Population Demographics

\begin{tabular}{lcc}
\hline \multicolumn{1}{c}{ Variables } & Men & Women \\
\hline No. of Patients & 80 & 80 \\
Age (yr) & $59.7 \pm 13.5$ & $55.3 \pm 12.9$ \\
Height $(\mathrm{cm})$ & $168.2 \pm 6.2^{*}$ & $155.5 \pm 5.9$ \\
Weight $(\mathrm{kg})$ & $65.7 \pm 9.2^{*}$ & $54.4 \pm 8.0$ \\
\hline
\end{tabular}

Values are given as number or mean $\pm \mathrm{SD}$. $* \mathrm{P}<0.05$.

on $3 \mathrm{D}$ images were smaller than those on $2 \mathrm{D}$ images. The differences in values of the AP diameters of the main bronchi between data measured on $3 \mathrm{D}$ and those measured on $2 \mathrm{D}$ images were also smaller than those of the TR diameters (Tables 2 and 3). The ratios, which were over or underestimated values on $3 \mathrm{D}$ images based on the values measured on 2D images, are described in Table 4. The degree of correlation of the length and diameter of the main bronchi between the data measured on $3 \mathrm{D}$ and on $2 \mathrm{D}$ images is described in Tables 2 and 3.

After choosing the left-sided DLTs based on the AP diameter measured $2 \mathrm{~cm}$ below the carina of the left main bronchus from the $3 \mathrm{D}$ and $2 \mathrm{D}$ images, respectively, the same sized DLTs were expected in $69 \%(55 / 80)$ of the men and $34 \%(27 / 80)$ of the women. 
Table 2. Measured Data, Difference in Values, and Degree of Correlation of the Main Bronchi between 2D and 3D Images in Men (mm)

\begin{tabular}{|c|c|c|c|c|c|}
\hline & $2 \mathrm{D}$ & $3 \mathrm{D}$ & $3 \mathrm{D}-2 \mathrm{D}$ & $\mathrm{R}$ values & $P$ values \\
\hline \multicolumn{6}{|l|}{ LMB } \\
\hline Length & $43.9 \pm 5.5$ & $48.2 \pm 4.5^{*}$ & $3.6(-7.1$ to 15.8$)$ & 0.470 & $<0.001$ \\
\hline AP diameter of $2 \mathrm{~cm}$ below the carina & $12.3 \pm 1.8$ & $13.0 \pm 1.6^{*}$ & $0.8(-4.0$ to 5.8$)$ & 0.520 & $<0.001$ \\
\hline TR diameter of $2 \mathrm{~cm}$ below the carina & $18.2 \pm 3.2$ & $13.5 \pm 2.2^{*}$ & $-4.3(-13.9$ to 2.5$)$ & 0.222 & $=0.047$ \\
\hline \multicolumn{6}{|l|}{$\mathrm{RMB}$} \\
\hline Length & $13.8 \pm 4.3$ & $13.4 \pm 3.4$ & $-0.4(-8.9$ to 8.4$)$ & 0.650 & $<0.001$ \\
\hline AP diameter of mid-point & $14.5 \pm 1.9$ & $15.4 \pm 1.7^{*}$ & $0.8(-3.7$ to 6.9$)$ & 0.371 & $<0.001$ \\
\hline TR diameter of mid-point & $19.8 \pm 3.2$ & $15.6 \pm 2.1^{*}$ & $-4.1(-14.2$ to 5.8$)$ & & NS \\
\hline
\end{tabular}

Values are mean \pm SD or median (range). 2D: two-dimensional image, 3D: three-dimensional image, LMB: left main bronchus, RMB: right main bronchus, AP: anteroposterior, TR: transverse, NS: not significant. 3D-2D: subtraction 2D from 3D. R values: correlation coefficient between 2D and 3D, $\mathrm{P}$ values: correlation significant between $2 \mathrm{D}$ and $3 \mathrm{D} .{ }^{*} \mathrm{P}<0.05$ by t-test between $2 \mathrm{D}$ and $3 \mathrm{D}$.

Table 3. Measured Data, Difference in Values, and Degree of Correlation of the Main Bronchi between 2D and 3D Images in Women (mm)

\begin{tabular}{|c|c|c|c|c|c|}
\hline & $2 \mathrm{D}$ & $3 \mathrm{D}$ & $3 \mathrm{D}-2 \mathrm{D}$ & $\mathrm{R}$ values & P values \\
\hline \multicolumn{6}{|l|}{ LMB } \\
\hline Length & $41.9 \pm 4.3$ & $43.6 \pm 3.6^{*}$ & $1.2(-5.8$ to 10.1$)$ & 0.650 & $<0.001$ \\
\hline AP diameter of $2 \mathrm{~cm}$ below the carina & $9.6 \pm 1.6$ & $10.4 \pm 1.5^{*}$ & $1.0(-2.5$ to 4.5$)$ & 0.586 & $<0.001$ \\
\hline TR diameter of $2 \mathrm{~cm}$ below the carina & $15.6 \pm 2.3$ & $10.1 \pm 2.0^{*}$ & $-5.7(-11.2$ to 1.0$)$ & & NS \\
\hline \multicolumn{6}{|l|}{ RMB } \\
\hline Length & $13.7 \pm 4.4$ & $11.8 \pm 2.7^{*}$ & $-1.3(-10.6$ to 5.3$)$ & 0.634 & $<0.001$ \\
\hline AP diameter of mid-point & $11.9 \pm 1.7$ & $13.3 \pm 1.4^{*}$ & $1.5(-5.1$ to 5.3$)$ & & NS \\
\hline TR diameter of mid-point & $18.4 \pm 3.7$ & $12.1 \pm 1.9^{*}$ & $-5.6(-16.1$ to 0.9$)$ & 0.261 & $=0.020$ \\
\hline
\end{tabular}

Values are mean \pm SD or median (range). 2D: two-dimensional image, 3D: three-dimensional image, LMB: left main bronchus, RMB: right main bronchus, AP: anteroposterior, TR: transverse, NS: not significant. 3D-2D: subtraction 2D from 3D. R values: correlation coefficient between 2D and $3 \mathrm{D}$, P values: correlation significant between $2 \mathrm{D}$ and $3 \mathrm{D} .{ }^{*} \mathrm{P}<0.05$ by t-test between $2 \mathrm{D}$ and $3 \mathrm{D}$.

Table 4. The Ratio of Over or Underestimated Values on 3D Images Based on the Measured Values on 2D Images

\begin{tabular}{|c|c|c|c|c|}
\hline & \multicolumn{2}{|c|}{ Overestimation } & \multicolumn{2}{|c|}{ Underestimation } \\
\hline & Men & Women & Men & Women \\
\hline \multicolumn{5}{|l|}{ LMB } \\
\hline Length & 79 & 61 & 21 & 39 \\
\hline AP diameter of $2 \mathrm{~cm}$ below the carina & 70 & 68 & 30 & 32 \\
\hline TR diameter of $2 \mathrm{~cm}$ below the carina & 7 & 1 & 93 & 99 \\
\hline \multicolumn{5}{|l|}{ RMB } \\
\hline Length & 49 & 30 & 51 & 70 \\
\hline AP diameter of mid-point & 64 & 79 & 36 & 21 \\
\hline TR diameter of mid-point & 14 & 2 & 86 & 98 \\
\hline
\end{tabular}

Values are \%. 2D: two-dimensional image, 3D: three-dimensional image, LMB: left main bronchus, RMB: right main bronchus, AP: anteroposterior, TR: transverse.

\section{Discussion}

Kauczor et al. [12] concluded that 3D, based on thin overlapping sections, visualized the normal airways down to the segmental level and could provide an accurate road map for the central airways. We investigated the degree of correlation between the values measured from the $3 \mathrm{D}$ and $2 \mathrm{D}$ images because $3 \mathrm{D}$ spiral chest CT scanning is rarely performed in clinical practice. In our study, a strong statistical correlation was not found; therefore, the values of $3 \mathrm{D}$ images cannot be estimated from the values measured using $2 \mathrm{D}$ images by the regression equation. However, correlation values of the length of the main bronchi and the AP diameter at $2 \mathrm{~cm}$ below the carina of the left main bronchus showed a moderate association (0.4-0.7) [13] in men and women.

The range of differences between the values of the AP diameter at $2 \mathrm{~cm}$ below the carina of the left main bronchus measured from the $3 \mathrm{D}$ and $2 \mathrm{D}$ images was great in our results. The difference of the outer diameters of the bronchial lumen in the size of the left-sided DLT (BronchoCath ${ }^{\mathrm{TM}}$, Mallinckrodt, St. Louis, 
MO, USA) was 0.5 to $0.9 \mathrm{~mm}$. To evaluate the clinical impact, we selected the left-sided DLT (BronchoCath ${ }^{\mathrm{TM}}$, Mallinckrodt, St. Louis, MO, USA) based on measured values on $3 \mathrm{D}$ and $2 \mathrm{D}$ images, respectively and then observed the degree of match. A good matching of $69 \%$ was observed in men, but a fair matching of $34 \%$ was observed in women. The left-sided DLT selected by $2 \mathrm{D}$ images might be used without major problems in men, but caution needs to be observed in women.

We tried to measure the exact length of the main bronchus for some slices in a way that there was an overlap on 2D images. The lengths of the left main bronchus measured on 3D images were higher than those measured on $2 \mathrm{D}$ images in men and women, but the lengths of the right main bronchus were smaller in women. These differences were due to an obliquely oriented main bronchi through the horizontal plane. More reliable values of the length might be obtained using 3D images [10]. So, the right-sided DLTs selected by measured bronchial diameter from $2 \mathrm{D}$ images should be used very carefully in women because they have a much smaller positioning margin of safety [14].

Studies for choosing the left-sided DLT based on the measured diameter of the left main bronchus measured on a 2D CT scan have been attempted [4,5]. Hannallah et al. [4] showed that an oversized DLT was selected for 5 out of 32 men (15.6\%) and 7 out of 18 women (38.9\%). Chow et al. [5] showed that an oversized DLT was selected for zero out of 12 men (0\%) and 3 out of 8 women $(37.5 \%)$. The mismatch rate was greater in women than in men. In anatomy, the right main bronchi pass inferolaterally and the left main bronchi pass inferoposterolaterally from the termination of the trachea to the hila of the lung. Since the main bronchus is oblique through the horizontal plane, the images appear elongated, and the ratio is consequently increased on $2 \mathrm{D}$ images [4]. The real value cannot be accurately measured in $2 \mathrm{D}$ CT scans. With recent advances in multi-slice spiral CT scanner technology, slice thicknesses can be less than $1 \mathrm{~mm}$. Whatever the original images, MPRs are usually indicated to visualize the intraluminal morphology of a tubular structure and can be obtained in real time [15]. Numerous reconstruction algorithms can be applied on a slab: shaded surface display (SSD), maximum intensity projection (MIP) or minimum intensity projection (mIP), volume rendering technique (VRT) [15]. VRT segmentation and rendering steps require much more computer power than SSD or MIP since all the data are used [16]. An advantage of VRT over SSD is that 3D-VRT is better than transverse images to analyze the morphology, site, and extent of the budding of a tubal structure [15]. The 3D-VRT method was used in our study.

Our study had some limitations. The adequate size of the leftsided DLT was not checked by directly intubating with the leftsided DLT, which was chosen by the AP diameter from the 3D and $2 \mathrm{D}$ images, respectively.

Our results showed that the lengths of the right and left main bronchi and their AP and TR diameters obtained from 3D images were not strongly correlated with those from 2D images. Therefore, a further study is needed to verify the superiority of $3 \mathrm{D}$ images in selecting the appropriate size of left-sided DLT.

\section{References}

1. Hannallah MS, Benumof JL, Bachenheimer LC, Mundt DJ. The resting volume and compliance characteristics of the bronchial cuff of left polyvinyl chloride double-lumen endobronchial tubes. Anesth Analg 1993; 77: 1222-6.

2. Hannallah M, Gomes M. Bronchial rupture associated with the use of a double-lumen tube in a small adult. Anesthesiology 1989; 71: 457-9.

3. Hannallah MS, Benumof JL, Ruttimann UE. The relationship between left mainstem bronchial diameter and patient size. J Cardiothorac Vasc Anesth 1995; 9: 119-21.

4. Hannallah M, Benumof JL, Silverman PM, Kelly LC, Lea D. Evaluation of an approach to choosing a left double-lumen tube size based on chest computed tomographic scan measurement of left mainstem bronchial diameter. J Cardiothorac Vasc Anesth 1997; $11: 168-71$.

5. Chow MY, Liam BL, Thng CH, Chong BK. Predicting the size of a double-lumen endobronchial tube using computed tomographic scan measurements of the left main bronchus diameter. Anesth Analg 1999; 88: 302-5.

6. Olivier P, Hayon-Sonsino D, Convard JP, Laloë PA, Fischler M. Measurement of left mainstem bronchus using multiplane CT reconstructions and relationship between patient characteristics or tracheal diameters and left bronchial diameters. Chest 2006; 130 : 101-7.

7. Curtin JJ, Innes NJ, Harrison BD. Thin-section spiral volumetric CT for the assessment of lobar and segmental bronchial stenoses. Clin Radiol 1998; 53: 110-5.

8. Rho WS, Sinn YS, Lee JS, Park CH, Park YC, Chung JY. The lengths of the left and right mainstem bronchi measured by computed tomography with minimum intensity projection in Korean adults. Korean J Anesthesiol 2006; 51: 426-9.

9. Tzeng YS, Hoffman E, Cook-Granroth J, Maurer R, Shah N, Mansour J, et al. Comparison of airway diameter measurements from an anthropomorphic airway tree phantom using hyperpolarized 3He MRI and high-resolution computed tomography. Magn Reson Med 2007; 58: 636-42.

10. Montaudon M, Berger P, de Dietrich G, Braquelaire A, Marthan R, Tunon-de-Lara JM, et al. Assessment of airways with three-dimensional quantitative thin-section CT: in vitro and in vivo validation. Radiology 2007; 242; 563-72. 
11. Edwards PD, Bull RK, Brown VS, Curtin J. Spiral CT optimization for measurement of bronchial lumen diameter using an experimental model. Br J Radiol 2000; 73: 715-9.

12. Kauczor HU, Wolcke B, Fischer B, Mildenberger P, Lorenz J, Thelen M. Three-dimensional helical CT of the tracheobronchial tree: evaluation of imaging protocols and assessment of suspected stenoses with bronchoscopic correlation. AJR Am J Roentgenol 1996; 167: 419-24.

13. Myles PS, Gin T. Statistical methods for anaesthesia and intensive care. Woburn, Butterworth-Heinemann. 2000, p 80.

14. Benumof JL, Partridge BL, Salvatierra C, Keating J. Margin of safety in positioning modern double-lumen endotracheal tubes. Anesthesiology 1987; 67: 729-38.

15. Remy J, Remy-Jardin M, Artaud D, Fribourg M. Multiplanar and three-dimensional reconstruction techniques in CT: impact on chest diseases. Eur Radiol 1998; 8: 335-51.

16. Johnson PT, Heath DG, Kuszyk BS, Fishman EK. CT angiography: thoracic vascular imaging with interactive volume rendering technique. J Comput Assist Tomogr 1997; 21: 110-4. 